\title{
PROMOCIÓN DE BIOMASA Y CONTENIDO DE AZÚCARES EN SORGO DULCE MEDIANTE ABONOS ORGÁNICOS Y MICORRIZA ARBUSCULAR
}

\author{
Arturo DÍAZ FANCO, Martín ESPINOSA RAMÍREZ y Flor Elena ORTIZ CHÁIREZ*
}

Instituto Nacional de Investigaciones Forestales Agrícolas y Pecuarias, Campo Experimental Río Bravo. Carretera Matamoros-Reynosa km 61, Apartado Postal 172, Ciudad Río Bravo, Tamaulipas, México, C.P. 88900

*Autor para correspondencia: ortiz.flor@inifap.gob.mx

(Recibido mayo 2015; aceptado noviembre 2015)

Palabras clave: gallinaza, Rhizophagus intraradices, Sorghum bicolor

\section{RESUMEN}

El sorgo dulce (Sorghum bicolor) es un cultivo que puede ser utilizado para producir azúcar, alcohol y biocombustible. La fertilización sintética ha cubierto las necesidades nutricionales del cultivo. Con el propósito de disminuir el uso de fertilizantes se evaluó la efectividad de la inoculación de la semilla con el hongo micorrízico arbuscular Rhizophagus intraradices (micorriza INIFAP) más la mitad de la dosis del fertilizante sintético convencional, la incorporación de gallinaza (Meyfer) ( $3 \mathrm{t} / \mathrm{ha}$ ), la fertilización sintética completa $(120 \mathrm{~kg} / \mathrm{ha}$ de $\mathrm{N} \mathrm{y} 40 \mathrm{~kg} / \mathrm{ha}$ de $\mathrm{P})$ y un tratamiento testigo absoluto sin fertilización ni inoculación. Las variables medidas fueron clorofila, biomasa de hojas, inflorescencia y tallo, así como el contenido de azúcares (grados Brix) de tallo en cada dos entrenudos. La aplicación de gallinaza o la inoculación de micorriza INIFAP más la mitad de la fertilización sintética, promovieron respuesta del sorgo dulce, en cantidad de biomasa y contenido de azúcares en el tallo. De modo que fue semejante al tratamiento que llevó la fertilización completa. Los resultados indican que la implementación de la inoculación micorrízica en sorgo dulce puede representar un manejo más amigable con el ambiente. La inoculación con el inoculante micorrízico INIFAP más la mitad de la fertilización sintética, constituyó el manejo de fertilización más económico (\$1217/ha) para la producción de sorgo dulce.

Key words: chicken manure, Rhizophagus intraradices, Sorghum bicolor

\begin{abstract}
Sweet sorghum (Sorghum bicolor) is a crop that can be used to produce sugar, alcohol and biofuel. Synthetic fertilization has covered the nutritional needs of the crop. With the purpose of reducing the use of fertilizers, the effectiveness of the seed inoculation with arbuscular mycorrhizal fungus Rhizophagus intraradices (micorriza INIFAP) plus half dose of conventional synthetic fertilizer, chicken manure (Meyfer) (3t/has), full synthetic fertilization (N $120 \mathrm{~kg} / \mathrm{ha}$ and P $40 \mathrm{~kg} / \mathrm{ha}$ ) and an absolute control without inoculation and fertilization treatment, was evaluated. The variables measured were chlorophyll, leaf, panicle and stem biomass, as well as stem sugar content (Brix degrees) in every two internodes. The application of chicken manure or inoculation of mycorrhiza INIFAP plus half the synthetic fertilization, promoted the response of sweet sorghum in terms of stem biomass and sugar content so that they were similar
\end{abstract}


to the treatment with full fertilization. The results indicate that the implementation of mycorrhizal inoculation in sweet sorghum may represent a more environmentally friendly management. Inoculation with mycorrhizal inoculant INIFAP plus half synthetic fertilization was the fertilization management with the lowest cost $(\$ 1217 / \mathrm{ha})$ for sweet sorghum production.

\section{INTRODUCCIÓN}

La reducción en la cantidad de recursos energéticos no renovables ha provocado en los últimos años incertidumbre e incremento en los precios de los combustibles. Aunado a esto, el calentamiento global debido al uso de recursos contaminantes es uno de los principales problemas que enfrenta la sociedad. Es por ello que resulta importante iniciar programas alternativos de producción de energía que reduzcan la contaminación y los gases con efecto invernadero que desestabilizan el clima (Prasad et al. 2007, Almodares y Hadi 2009). Las plantas cultivadas son la mejor fuente de energía renovable que pueden ser utilizadas para la producción de biocombustibles. Brasil y Estados Unidos son los países líderes mundiales en la producción de etanol, el primero tiene como fuente el cultivo de caña de azúcar y el segundo el maíz. Sin embargo, la expansión de la industria del etanol dependerá de la eficiencia en la producción. En particular la caña de azúcar y el maíz son cultivados en condiciones de altos insumos, en especial agua y fertilizantes (Calviño y Messing 2012).

El caso del sorgo dulce (Sorghum bicolor), presenta ventajas comparativas, no compite con cultivos de granos y tiene bajos costos de producción, requiere $36 \%$ menos nitrógeno que el maíz para lograr rendimientos similares de etanol (Ratnavathi et al. 2011). Es una gramínea de fotosíntesis $\mathrm{C}_{4}$, tolerante a la sequía, de rápido crecimiento, que acumula grandes cantidades de azúcares fermentables en el tallo y de biomasa, posee amplia adaptabilidad y tolera condiciones adversas de producción, además su siembra es factible en áreas no aptas para otros cultivos (Ratnavathi et al. 2011, Calviño y Messing 2012). Por lo anterior se puede utilizar para la producción de azúcar refinada, alcohol y gasolina, entre otros (House et al. 2002, Lemus y Parrish 2009, Ratnavathi et al. 2011). Es importante señalar que el uso de biocombustibles produce energía limpia y eficiente (Prasad et al. 2007, Almodares y Hadi 2009). Asimismo, se ha percibido la necesidad de implementar estrategias agronómicas que permitan, entre otras cosas, mitigar los efectos adversos sobre el suelo, disminuir la tasa de uso de fertilizantes sintéticos y aumentar las ganancias por área cultivada (Adesemoye y Kloepper 2009, Carbajal y Mera 2010).

La práctica de biofertilización o fertilización biológica se divide en dos aspectos: a) la adición al suelo de restos de materia orgánica a través de abonos orgánicos, aguas residuales, biosólidos, residuos animales (estiércoles) y vegetales, composta, vermicomposta, entre otros y b) la inoculación de bioinoculantes, conformados por microorganismos (hongos y bacterias) que colonizan el sistema radical de las plantas con potencial biofertilizante (Hue y Silva 2000, Aguado 2012). Los estiércoles tienen el potencial de ser utilizados como una fuente económica de nutrimentos y de gran eficiencia en la nutrición de los cultivos, aunque según sus procedencias, muestran grandes diferencias en cuanto a los nutrimentos que contienen. De los diferentes tipos de estiércoles, la gallinaza y el de cerdo son los más ricos desde el punto nutrimental y de mayor liberación de nutrimentos en el primer año. Mientras que, los estiércoles más pobres son el vacuno y el equino (Hue y Silva 2000).

En particular, los hongos micorrízicos arbusculares (HMA), como parte de su actividad simbiótica manifiestan diferentes mecanismos que inducen una mayor exploración del suelo a través de las hifas, disminuyen los efectos de condiciones abióticas adversas para la planta, producen fitohormonas que estimulan su crecimiento, facilitan la absorción de nutrimentos, producen glomalina que adhiere las partículas del suelo e inducen acción protectora contra algunos fitopatógenos del suelo. Estas cualidades han demostrado la importancia de las interacciones entre las asociaciones HMA-planta, con capacidad de obtener beneficios agronómicos eficientes (Smith y Read 2008, Nadeem et al. 2014).

Las necesidades de fertilización y dosis en sorgo dulce han sido ampliamente documentadas (Wiedenfel 1984, Almodares et al. 1996, Rego et al. 2003, Montes et al. 2013). Para la región norte de Tamaulipas, la dosis de fertilización recomendada para sorgo de grano y dulce es de 120-40-00 (Salinas 2006, Montes et al. 2010b). Sin embargo, esta práctica trae consigo serias repercusiones económicas (representa un insumo de alto costo) y ambientales. Se estima 
que entre 10 y $40 \%$ de los fertilizantes aplicados son utilizados por las plantas (Duxbury 1994), el resto constituyen contaminantes de cuerpos de agua provenientes del proceso de lixiviación. Además, aportan emisiones de dióxido de carbono $\left(\mathrm{CO}_{2}\right)$ y óxidos de nitrógeno a la atmósfera, gases que originan el efecto invernadero (Walker 1990, Crewsa y Peoples 2004). Estos riesgos de contaminación también los pueden generar los abonos orgánicos aplicados en los cultivos (Cooperband 2002, Bouajila y Sanna 2011), aunque podrían representar beneficios como mejorar la estructura del suelo, aumentar la biodiversidad y biomasa microbiana, además de reducir los costos de producción. Por lo anterior, un enfoque alterno es utilizar bajas cantidades de abonos orgánicos (Álvarez et al. 2010).

La fertilización biológica representa una importante alternativa tecnológica que podría coadyuvar en la producción de sorgo dulce, particularmente en la promoción de la cantidad de azúcares y de biomasa. Diferentes estudios sobre biofertilización en el agro mexicano han demostrado beneficios al incrementar la productividad de granos (Aguado 2012, Grageda et al. 2012) y en específico con sorgo para grano (Díaz et al. 2011). No obstante, se desconoce la influencia que pudieran tener los abonos orgánicos y los microorganismos benéficos en la producción de biomasa (azúcares) en el sorgo dulce. Díaz et al. (2015) demostraron similitudes en características de la planta y la productividad de sorgo para grano, cultivado con fertilización convencional (120-40-00) respecto al cultivado con la mitad de la fertilización sintética más la inoculación de la especie de HMA, Rhizophagus intraradices (micorriza INIFAP). Por lo anterior, se realizó un estudio con el propósito de conocer la productividad de sorgo dulce en función de la biomasa y del contenido de azúcares, mediante la adición de gallinaza y la inoculación de micorriza INIFAP con fertilización sintética reducida.

\section{MATERIALES Y MÉTODOS}

Sitio experimental. El experimento se desarrolló en terrenos del Campo Experimental Río Bravo
(CERIB) del Instituto Nacional de Investigaciones Forestales, Agrícolas y Pecuarias (INIFAP), Río Bravo, Tamaulipas, México. $\left(25^{\circ} 57^{\prime} 54^{\prime \prime} \mathrm{N}, 98^{\circ} 01^{\prime} 05^{\prime}\right.$ " O, $25 \mathrm{msnm}$ ), durante el ciclo otoño-invierno 20132014 y en condiciones de riego. Previo a la siembra, se hizo un muestreo de suelo a profundidad 0-30 $\mathrm{cm}$ y un posterior análisis de $\mathrm{pH}$, M.O., C.E., N, P, $\mathrm{K}$, y textura (Cuadro I). Los análisis de suelo se realizaron en el Laboratorio de Agua-Suelo y Planta del CERIB. El pH se determinó en solución acuosa (1:2), la conductividad eléctrica (C.E.) mediante conductímetro con pasta de saturación, la materia orgánica (M.O.) se midió con dicromato de potasio (Walkely y Black), el $\mathrm{NO}_{3}-\mathrm{N}$ se determinó mediante ácido acetilsalicílico, el $\mathrm{P}$ disponible se midió con el método de Olsen y el K intercambiable mediante acetato de amonio.

Manejo experimental. La siembra se estableció el 19 de febrero y la variedad de sorgo dulce utilizada fue 'Candy'. Los tratamientos evaluados fueron: a) fertilización sintética recomendada o testigo fertilizado, 120 y $40 \mathrm{~kg} / \mathrm{ha}$ de $\mathrm{N}$ y P, respectivamente (Montes et al. 2010b), b) mitad de fertilización sintética más inoculación de la semilla con micorriza INIFAP (HMA) con dosis de $0.5 \mathrm{~kg} / \mathrm{ha}$ (8 $\mathrm{kg}$ de semilla), 60-20-00 + HMA (Díaz et al. 2015), c) gallinaza a razón de 3 t/ha y d) testigo absoluto. Los tratamientos se distribuyeron en un diseño de bloques completos aleatorios con cuatro repeticiones. La parcela experimental consistió de cuatro surcos de $0.81 \mathrm{~m}$ de ancho y $6 \mathrm{~m}$ de longitud, la densidad de plantas se ajustó a 145000 plantas/ha. La micorriza INIFAP está conformada por el HMA Rhizophagus intraradices (Sin. Glomus intraradices), con $\geq 60$ esporas/g. Se utilizó gallinaza procesada y deshidratada [Meyfer ${ }^{\circledR}$ (4.64\% N total, $5.42 \% \mathrm{P}, 3.13 \% \mathrm{~K}, 338 \mathrm{mg} / \mathrm{kg}$ $\mathrm{Cu}, 6588 \mathrm{mg} / \mathrm{kg} \mathrm{Fe}, 799 \mathrm{mg} / \mathrm{kg} \mathrm{Mn}, 568 \mathrm{mg} / \mathrm{kg} \mathrm{Zn}$, $42.1 \%$ M.O.)], la cual se incorporó a un lado del surco el 17 de enero. El fertilizante, a base de urea y fosfato monoamónico, se aplicó de la misma forma pero al momento de la siembra. La mitad del $\mathrm{N}$ se agregó en la siembra y la otra a los 36 días después de la misma. Los riegos fueron en presiembra y dos de auxilio. Para el manejo agronómico del cultivo se siguieron las indicaciones locales (Montes et al. 2010b).

CUADRO I. PROPIEDADES FÍSICAS Y QUÍMICAS DEL SUELO PREVIAS AL ESTABLECIMIENTO DEL EXPERIMENTO

\begin{tabular}{cccccccccc}
\hline $\begin{array}{c}\text { M.O. } \\
(\%)\end{array}$ & $\mathrm{pH}$ & C.E. $(\mathrm{dS} / \mathrm{m})$ & $\begin{array}{c}\text { Arena } \\
(\%)\end{array}$ & $\begin{array}{c}\text { Arcilla } \\
(\%)\end{array}$ & $\begin{array}{c}\text { Limo } \\
(\%)\end{array}$ & Textura & $\begin{array}{c}\mathrm{N}^{2} \mathrm{NO}_{3} \\
(\mathrm{mg} / \mathrm{kg})\end{array}$ & $\begin{array}{c}\mathrm{P} \\
(\mathrm{mg} / \mathrm{kg})\end{array}$ & $\begin{array}{c}\mathrm{K} \\
(\mathrm{mg} / \mathrm{kg})\end{array}$ \\
\hline 1.70 & 7.61 & 0.97 & 37.2 & 43.3 & 19.4 & Arcilla & 21.1 & 13.9 & 1208 \\
\hline
\end{tabular}

M.O., materia orgánica, C.E., conductividad eléctrica 
Variables evaluadas. De los surcos centrales se tomaron siete plantas por parcela, en ellas se midieron: a) el índice de clorofila (en unidades SPAD con medidor digital Minolta SPAD 502 ${ }^{\circledR}$ ) del centro de las hojas y del tercio superior de las plantas en estado de floración, con 10 lecturas por parcela, b) el contenido de azúcares en tallo en estado de grano lechoso-masoso (en grados Brix con refractómetro), cuantificado cada dos entrenudos (Montes et al. 2010a) y c) el peso en biomasa fresca de hojas, tallo e inflorescencia por planta.

Análisis estadístico. Los datos de las variables de estudio se sometieron a análisis de varianza, la separación de medias fue mediante la prueba de Tukey con un nivel de confianza de $95 \%$. Estos análisis se realizaron a través del programa SAS (versión 8.1).

\section{RESULTADOS Y DISCUSIÓN}

Los tratamientos de fertilización biológica y sintética impactaron significativamente en el índice de clorofila y en la biomasa aérea del sorgo dulce. Diferencias significativas $(p<0.05)$ se observaron en el contenido de clorofila, de manera que todos los tratamientos de fertilización superaron al testigo absoluto, los cuales promediaron 40.8 (unidades SPAD). El contenido de pigmentos fotosintéticos es un indicador del estatus nutrimental de la planta (Piekielek y Fox 1992). Asimismo, el peso de hojas, de panojas y total de las plantas, aumentaron significativamente con los tratamientos evaluados comparados con el testigo. Adicionalmente el mayor peso del tallo fue con 60-20-00 + HMA y diferente al testigo. En la producción de biomasa por hectárea, el testigo alcanzó 50.7 t/ha, mientras que el resto de los tratamientos de fertilización promediaron 57.3 t/ha (Cuadro II). Lo anterior refleja el impacto benéfico que tuvieron los tratamientos en la biomasa desglosada en cada componente de la planta, lo cual explica la biomasa total producida, aunque la del tallo es la característica relevante del cultivo para la producción de biocombustible (Hernández et al. 2013, Montes et al. 2013).

Para el caso del contenido de azúcares en el tallo, los valores fluctuaron de 14.6 a $19.5^{\circ}$ Brix y se encuentran en los rangos obtenidos en otros estudios (Montes et al. 2010a, b, Ratnavathi et al. 2011). En general, las mayores concentraciones se registraron en los entrenudos 4, 6 y 8. En los entrenudos 2, 6 y 8 no se observaron diferencias significativas en ${ }^{\circ}$ Brix. Aunque en particular, para el entrenudo 4 y el promedio de los cuatro, los tratamientos de fertilización superaron de manera similar $(\mathrm{p}<0.05)$ al testigo absoluto. El testigo registró 16.5, mientras que el promedio del resto de los tratamientos fue de $17.5^{\circ} \mathrm{Brix}$ (Cuadro III). En un estudio de sorgo dulce con diferentes densidades de población, Valadez et al. (2011) obtuvieron la mayor concentración de azúcares $\left(15.4^{\circ}\right.$ Brix $)$ cuando el cultivo se sembró a densidad de 200-210 mil plantas/ha. Respecto a los efectos que tiene la fertilización biológica en la producción de sorgo dulce no existen antecedentes. Sin embargo, los resultados de Lopes et al. (2012) registrados en caña de azúcar (Saccharum officinarum), demostraron variaciones de efectividad de diferentes inoculantes microbianos para altura de planta, diámetro de tallo $\mathrm{y}^{\circ}$ Brix.

Es importante destacar que la biomasa de tallo y la producción de azúcares son variables no asociadas $(\mathrm{r}=0.201)$ y que desde el punto de vista comercial el componente importante es el peso de biomasa de tallo por unidad de superficie, equivalente a $73 \%$ de la biomasa total de la planta. Según Montes et al. (2013), el resto de los componentes, hojas y panojas, constituyen subproductos adicionales que se pueden comercializar como materia seca (pacas).

CUADRO II. RESPUESTA DE CLOROFILA Y BIOMASA DEL SORGO DULCE 'CANDY' A LA FERTILIZACIÓN SINTÉTICA Y BIOLÓGICA, CAMPO EXPERIMENTAL RÍO BRAVO, TAMAULIPAS

\begin{tabular}{|c|c|c|c|c|c|c|}
\hline \multirow[t]{2}{*}{ Fertilización } & \multirow{2}{*}{$\frac{\text { Clorofila }}{\text { (unidades SPAD) }}$} & \multicolumn{4}{|c|}{ Biomasa (g)/planta } & \multirow{2}{*}{$\frac{\text { Biomasa }}{(\mathrm{t} / \mathrm{ha})}$} \\
\hline & & Hoja & Panoja & Tallo & Total & \\
\hline \multicolumn{7}{|c|}{ 60-20-00 + Rhizophagus } \\
\hline Gallinaza (3 t/ha) & $40.5 \mathrm{a}$ & $50.2 \mathrm{a}$ & $53.0 \mathrm{ab}$ & $290.2 \mathrm{ab}$ & $393.4 \mathrm{a}$ & $57.0 \mathrm{a}$ \\
\hline $120-40-00$ & $40.6 \mathrm{a}$ & $52.1 \mathrm{a}$ & $58.4 \mathrm{a}$ & $281.5 \mathrm{ab}$ & $392.0 \mathrm{a}$ & $56.8 \mathrm{a}$ \\
\hline Testigo & $37.3 \mathrm{~b}$ & $46.0 \mathrm{~b}$ & $47.1 \mathrm{~b}$ & $257.2 \mathrm{~b}$ & $350.3 \mathrm{~b}$ & $50.7 \mathrm{~b}$ \\
\hline
\end{tabular}

*Valores con misma letra en columna no difieren significativamente (Tukey, $\mathrm{p} \leq 0.05$ ) 
CUADRO III. INFLUENCIA DE LA FERTILIZACIÓN SINTÉTICA Y BIOLÓGICA EN ${ }^{\circ}$ BRIX, MEDIDOS EN CUATRO ENTRENUDOS DEL TALLO DE SORGO DULCE 'CANDY', CAMPO EXPERIMENTAL RÍO BRAVO, TAMAULIPAS

\begin{tabular}{lccccc}
\hline \multirow{2}{*}{ Fertilización } & \multicolumn{5}{c}{${ }^{\circ}$ Brix en los entrenudos/planta } \\
\cline { 2 - 6 } & 2 & 4 & 6 & 8 & Promedio \\
\hline $60-20-00+$ Rhizophagus & & & & & \\
intraradices & $15.5 \mathrm{a}^{*}$ & $18.2 \mathrm{a}$ & $18.8 \mathrm{a}$ & $17.3 \mathrm{a}$ & $17.4 \pm 1.4 \mathrm{a}$ \\
Gallinaza $(3 \mathrm{t} / \mathrm{ha})$ & $15.1 \mathrm{a}$ & $18.6 \mathrm{a}$ & $18.9 \mathrm{a}$ & $17.5 \mathrm{a}$ & $17.5 \pm 1.7 \mathrm{a}$ \\
120-40-00 & $15.6 \mathrm{a}$ & $18.1 \mathrm{a}$ & $19.5 \mathrm{a}$ & $18.2 \mathrm{a}$ & $17.8 \pm 1.6 \mathrm{a}$ \\
Testigo & $14.6 \mathrm{a}$ & $16.8 \mathrm{~b}$ & $18.0 \mathrm{a}$ & $16.9 \mathrm{a}$ & $16.5 \pm 1.4 \mathrm{~b}$ \\
\hline
\end{tabular}

*Valores con misma letra en columna no difieren significativamente (Tukey, $\mathrm{p} \leq 0.05$ )

La mitad de la fertilización sintética más la inoculación a la semilla con micorriza INIFAP (6020-00 + HMA) o la adición de $3 \mathrm{t} / \mathrm{ha}$ de gallinaza, manifestaron una respuesta semejante en relación con el testigo fertilizado con 120-40-00 y superior al testigo absoluto. Por lo anterior resulta evidente que los valores de clorofila, de biomasa aérea y contenido de azúcares en el tallo, con la combinación 60-20-00 + HMA o gallinaza, mostraron una respuesta similar al testigo fertilizado. Dado el impacto que tuvo la asociación entre la fertilización sintética-micorriza o gallinaza en el sorgo dulce, será conveniente estudiar diferentes alternativas de fertilización biológica que puedan aportar mayor eficiencia en la producción y reducir o remplazar la fertilización mineral. La respuesta agronómica observada en este estudio con la fertilización biológica también se ha registrado en otros cultivos. Resultados semejantes se han obtenido con sorgo para grano, Díaz et al. (2015) determinaron que con la mitad del fertilizante sintético más la inoculación de micorriza INIFAP, se obtuvo similar longitud de panoja, granos/panoja, peso de 100 granos y rendimiento de grano, comparado con la fertilización completa (120-40-00). En otros cultivos también se ha informado sobre el potencial que tiene la bioinoculación para reducir las dosis de fertilización. Por ejemplo, Sharma et al. (2011) reportaron el mayor rendimiento de trigo (Triticum aestivum) con la inoculación de $R$. intraradices combinada con el $75 \%$ de N-P-K adicionado al suelo. Carpio et al. (2005) indicaron que las mejores características para el mercado de Ipomoea carnea ssp. fistulosa, así como la mayor absorción de N, P y K, ocurrió tanto con el inoculante BioterraPLUS ${ }^{\circledR}$ y la adición de 50 $\%$ de N-P-K en el suelo, como con la fertilización al $100 \%$. Los HMA juegan un papel destacado en la fertilidad ya que modifican las características químicas del suelo, las cuales están sujetas a las interacciones entre suelo-planta-HMA, lo que da como resultado su capacidad amortiguadora de fertilidad (Baera 1991). Marschner y Dell (1994) y Morgan et al. (2005) señalan que cuando se establece la interacción planta-HMA, el hongo incrementa la asimilación de nutrimentos con aportaciones de hasta $25 \%$ de N, $80 \%$ de P, $10 \%$ de K, $25 \%$ de Zn y $60 \%$ de $\mathrm{Cu}$, entre otros. En función al uso continuo del inoculante micorrízico, no están claras las consecuencias ecológicas en la diversidad de HMA nativos (por competencia o desplazamiento), debido al efecto de la introducción de cepas de HMA. Aunque Antunes et al. (2009) demostraron que la introducción de un inoculante comercial con base en $R$. intraradices interactuó directa o indirectamente de forma sinérgica con el suelo (perturbado o no perturbado) y sin impacto negativo en la competencia o estructura de las comunidades de HMA nativos.

Es probable que la aportación de la gallinaza en el sorgo dulce se deba a los efectos favorables que proporciona al suelo, ya que como abono orgánico se considera imprescindible en el uso y manejo del suelo para mejorar y mantener su componente orgánico, la fertilidad física, química y biológica, en síntesis, su calidad y productividad (Cooperband 2002, Sharma et al. 2012). Se ha demostrado que la mayoría de los cultivos manifiestan una clara respuesta a la aplicación de abonos orgánicos, en particular en suelos sometidos a cultivos de forma consistente, debido a que aportan casi todos los nutrimentos que las plantas necesitan, comparados con la fertilización sintética. Aunque estos abonos orgánicos contienen menores nutrimentos, la disponibilidad de éstos es constante para la planta por la mineralización gradual a que están sometidos (Carbajal y Mera 2010, Bouajila y Sanaa 2011). La aplicación de materia orgánica también aumenta la 
actividad enzimática, las fosfatasas y la ureasa tienen particular relevancia por su trascendencia en los ciclos biogeoquímicos y de nutrición (Álvarez et al. 2010). No obstante, en general el uso de los abonos orgánicos tiene implicaciones como contaminante ambiental. Su impacto incluye: i) la lixiviación de los elementos contenidos en los abonos orgánicos origina la contaminación de aguas subterráneas, ii) inducen el efecto invernadero debido a las emisiones de dióxido de carbono y óxidos de nitrógeno a la atmósfera, iii) pueden incrementar las poblaciones de malezas en los cultivos y iv) con el tiempo ocasionan la acumulación de sales ( $\mathrm{Na}$ ) y metales pesados en el suelo (Carbajal y Mera 2010, Bouajila y Sanaa 2011). De aquí la conveniencia de utilizar preferentemente bajas cantidades de abonos orgánicos. En las condiciones del estudio, $3 \mathrm{t} / \mathrm{ha}$ de gallinaza procesada fueron suficientes para obtener resultados semejantes a los de fertilización convencional. Por el contrario, López et al. (2001) y Fortis et al. (2009) requirieron de 20 a $30 \mathrm{t} / \mathrm{ha}$ de composta para sustituir la fertilización inorgánica en maíz.

En relación con los costos de los insumos, el precio estimado de la dosis de 120-40-00 es de \$2334/ ha, de $\$ 4500 /$ ha para gallinaza y de $\$ 50 /$ ha para la micorriza INIFAP, por lo que el tratamiento más económico fue 60-20-00 + HMA (\$1217/ha). Los resultados demostraron que la fertilización biológica, puede representar una estrategia agronómica que permite mejorar la eficiencia del cultivo, disminuir o suplir las necesidades de fertilizantes sintéticos, reducir los costos de producción e inducir menor impacto negativo en la agroecología, tal como lo señalaron Cooperband (2002), Plenchette et al. (2005) y Sharma et al. (2012). Calvo et al. (2013) enfatizaron que para poder reducir las emisiones de $\mathrm{N}_{2} \mathrm{O}$ asociadas con la aplicación de fertilizantes nitrogenados, es importante considerar el uso de bioinoculantes como parte del manejo agronómico.

\section{CONCLUSIONES}

La aplicación de 3 t/ha de gallinaza o la inoculación de micorriza INIFAP más la mitad de la fertilización sintética, estimuló una respuesta del sorgo dulce, en función a la biomasa y el contenido de azúcares en el tallo, semejante al tratamiento que llevó la fertilización total o convencional. La inoculación de micorriza INIFAP más la mitad de la fertilización sintética, representó el manejo más económico (\$1217/ha) al compararla con el resto de los tratamientos.

\section{AGRADECIMIENTOS}

Se agradece el apoyo recibido de la Secretaría de Agricultura, Ganadería, Desarrollo Rural, Pesca y Alimentación (SAGARPA) de México a través del proyecto "Investigación y transferencia de tecnología sobre el empleo de biofertilizantes y abonos orgánicos en la agricultura". Al Sr. Juan Olvera Martínez, por la asistencia en el trabajo de campo.

\section{REFERENCIAS}

Adesemoye A.O. y Kloepper J.W. (2009). Plant-microbes interactions in enhanced fertilizer-use efficiency. Appl. Microbiol. Biotechnol. 85, 1-12.

DOI: $10.1007 / \mathrm{s} 00253-009-2196-0$.

Aguado S.G. (2012). Introducción al uso y manejo de los biofertilizantes en la agricultura. Instituto Nacional de Investigaciones Forestales, Agrícolas y Pecuarias/Secretaría de Agricultura, Ganadería, Desarrollo Rural, Pesca y Alimentación. Ciudad de México, México, 296 pp.

Álvarez S.J.D., Gómez V.D., León M.S. y Gutiérrez M.F. (2010). Manejo integrado de fertilizantes y abonos orgánicos en el cultivo de maíz. Agrociencia 44, 575-586.

Almodares A., Aghamiri A. y Sepahi A. (1996). Effects of the amount and time of nitrogen fertilization on carbohydrate contents of three sweet sorghum cultivars. Ann. Plant Physiol. 10, 56-60.

Almodares A. y Hadi M.R. (2009). Production of bioethanol from sweet sorghum: A review. Afr. J. Agr. Res. 4, 772-780.

Antunes M.P., Koch A., Dunfield K., Hart M., Downing A., Rilling M. y Klironomos J. (2009). Influence of commercial inoculation with Glomus intraradices on the structure and functioning of an AM fungal community from an agricultural site. Plant Soil 317, 257-266.

Baera J.M. (1991). Vesicular-arbuscular mycorrhizae as modifiers of soil fertility. Adv. Soil Sci. 15, 1-40.

Bouajila K. y Sanaa M. (2011). Effects of organic amendments on soil physic-chemical and biological properties. J. Matter Environ. Sci. 2, 485-490.

Calviño M. y Messing J. (2012). Sweet sorghum as a model system for bioenergy crops. Curr. Opin. Biotechnol. 23, 323-329. DOI: 10.1016/j.copbio.2011.12.002.

Calvo P., Watts D.B., Ames R.N., Kloepper J.W. y Torbert H.A. (2013). Microbial-based inoculants impact nitrous oxide emissions from an incubated soil medium containing urea fertilizers. J. Environ. Qual. 42, 704712. DOI: $10.2134 /$ jeq2012.0300

Carbajal M.J y Mera B.A. (2010). Fertilización biológica: técnicas de vanguardia para el desarrollo agrícola sostenible. Producción 5, 77-96. 
Carpio A.L., Davies T.F. y Arnold A.M. (2005). Arbuscular mycorrhizal fungi, organic and inorganic controlledrelease fertilizers: Effect on growth and leachate of container-grown bush morning glory (Ipomoea carnea ssp. fistulosa) under high production temperatures. J. Amer. Soc. Hort. Sci. 130, 131-139

Cooperband L. (2002). Building soil organic matter with organic amendments. University of WisconsinMadison, Wisconsin, EUA, 13 pp.

Crewsa T.E. y Peoples M.B. (2004). Legume versus fertilizer sources of nitrogen: ecological tradeoffs and human needs. Agr. Ecosyst. Environ. 102, 279-297.

Díaz F.A, Pecina Q.V, Montes G.N, Jacques H.C. y Garza C.I. (2011). Impacto de inoculantes microbianos en sorgo cultivado bajo déficit de humedad en el suelo. En: Retos de la investigación del agua en México (U. Oswald, Ed). Universidad Nacional Autónoma de México-Consejo Nacional de Ciencia y Tecnología. Ciudad de México, México, pp. 273-282.

Díaz F.A., Ortiz C.F. y Gálvez L.D. (2015). Bioinoculación y fertilización química reducida asociados con el crecimiento de planta y productividad de sorgo. Rev. Inter. Cont. Ambie. 31, 245-252.

Fortis H.M., Leos R.J., Preciado R.P., Orona C.I., García S.J., García H.V. y Orozco V.J. (2009). Aplicación de abonos orgánicos en la producción de maíz forrajero con riego por goteo. Terra Latinoamer. 27, 329-336.

Grageda C.O., Díaz F.A., Peña C.J. y Vera N.J. (2012). Impacto de los biofertilizantes en la agricultura. Rev. Mex. Cien. Agr. 3, 1261-1274.

Hernández M.M., Borodanenko A., Montes H.S., Pecina Q.V., Montoya C.L., Acosta N.M. y Rivas G.O. (2013). Manual técnico de producción de cultivos bioenergéticos. Campo Experimental Bajío, Instituto Nacional de Investigaciones Forestales, Agrícolas y Pecuarias. Reporte Técnico No. 2. Celaya, Guanajuato, México, $121 \mathrm{pp}$.

House H.R., Gómez, M., Murty O.S., Sun Y. y Verma B.N. (2002). Development of some agricultural industries in several African and Asian countries. John Wiley and Sons, Nueva York, EUA, 490 pp.

Hue N.V. y Silva J.A. (2000). Organic soil amendments for sustainable agriculture. En: Plant nutrient management (A. Silva y R. Uchida, Eds.). University of Hawaii. Hawaii, EUA, pp. 133-144.

Lemus R. y Parrhis D.J. (2009). Herbaceous crops with potential for biofuel production in the USA. Perspectives in agriculture science, nutrition and natural resourses. Food and Agriculture Organization, Publ. 57. Washington, EUA, $89 \mathrm{pp}$.

Lopes R.V., Bespalhok F.J., Araujo L. M., Vieira R.F., Daros E. y Augusto O.R. (2012). The selection of sugarcane that display better associations with plant growth promoting rhizobacteria. J. Agr. 11, 43-54. DOI: 10.3923/ja.2012.43.52.

López M.J., Díaz E.A., Martínez R.E. y Valdez C.R. (2001). Abonos orgánicos y su efecto en propiedades físicas y químicas del suelo y rendimiento de maíz. Terra Latinoamer. 19, 293-299.

Marschner H. y Dell B. (1994). Nutrient uptake in mycorrhizal symbiosis. Plant Soil 159, 89-102.

Montes G.N, Pecina Q.V, Cisneros L.M. y García G.M. (2010a). RB Cañero: Sorgo dulce para la producción de etanol. Folleto Técnico No. 43. Campo Experimental Río Bravo. Instituto Nacional de Investigaciones Forestales, Agrícolas y Pecurias, Río Bravo, Tamaulipas, México, $31 \mathrm{pp}$.

Montes G.N, Salinas G.J, González J.A., Loredo P.R. y Díaz P.G. (2010b). Guía técnica de producción de sorgo dulce [Sorghum bicolor (L.) Moench] en Tamaulipas. Reporte Técnico No. 49. Campo Experimental Río Bravo. Instituto Nacional de Investigaciones Forestales, Agrícolas y Pecuarias. Río Bravo, Tamaulipas, México, $35 \mathrm{pp}$.

Montes G.N., Vargas V.E., Salinas G.J., Espinosa R.M. y Loredo P.R. (2013). Tecnología de producción de sorgo dulce para la elaboración de bioethanol en Tamaulipas. Folleto 21. Campo Experimental Río Bravo. Instituto Nacional de Investigaciones Forestales, Agrícolas y Pecuarias. Río Bravo, Tamaulipas, México, $26 \mathrm{pp}$.

Morgan J.A., Bending G.D. y White P.J. (2005). Biological cost and benefits to plant-microbe interactions in the rhizosphere. J. Exp. Bot. 56, 1729-1739.

DOI: $10.1093 / \mathrm{jxb} / \mathrm{eri2} 205$.

Nadeem S.M., Ahmad M., Zahir A., Javaid A. y Ashraf M. (2014). The role of mycorrhizae and plant growth promoting rhizobacteria (PGPR) in improving crop productivity under stressful environments. Biotechnol. Adv. 32, 429-448.

DOI: 10.1016/j.biotechadv.2013.12.005.

Piekielek W.P. y Fox R.H. (1992). Use of chlorophyll meter to predict sidedress nitrogen requirements for maize. Agron. J. 84, 59-65.

Plenchette C., Dauphin C.C., Maynard J.M. y Fortin J.A. (2005). Managing arbuscular mycorrhizal fungi in cropping systems. Can. J. Plant Sci. 85, 31-40.

DOI: 10.4141/P03-159.

Prasad S., Singh A., Jain N. y Joshi H.C. (2007). Ethanol production from sweet sorghum syrup for utilization as automotive fuel in India. Energy Fuels 21, 2415-2420. DOI: $10.1021 / \mathrm{ef060328z}$.

Ratnavathi C.V., Kalyana S., Komala V.V., Chavan U.D. y Patil J.V. (2011). Sweet sorghum as feedstock for biofuel production: A review. Sugar Tech. 13, 399-407. DOI: $10.1007 / \mathrm{s} 12355-011-0112-2$. 
Rego T.J., Rao V.N., Seeling B., Pardhasaradhi G. y Rao J.V. (2003). Nutrient balances a guide to improving sorghum and ground based dry land cropping systems in semi-arid tropical India. Field Crop Res. 81, 53-68.

Salinas G.J. (2006). Fertilización del sorgo en el norte de Tamaulipas. Reporte Técnico No. 1. Campo Experimental Río Bravo. Instituto Nacional de Investigaciones Forestales, Agrícolas y Pecuarias. Río Bravo, Tamaulipas, México, $30 \mathrm{pp}$.

Sharma M., Reddy U. y Adholeya A. (2011). Response of arbuscular mycorrhizal fungi on wheat (Triticum aestivum) grown conventionally and on beads in sandy loam soil. Indian J. Microbiol. 3, 384-389.

DOI: $10.1007 / \mathrm{s} 12088-011-0134-1$.

Sharma S., Gupta R., Dugar G. y Srivastava A. (2012). Impact of application of biofertilizers on soil structure and resident microbial community structure and function. En: Bacteria in agrobiology: Plant probiotics (D.K. Maheshwari, Ed.). Hauz Khas, Nueva Delhi, India, pp. 65-79.

Smith G.S. y Read D.J. (2008). Mycorrhizal symbiosis. 3a. ed. Academic Press. Londres, Inglaterra, 750 pp.

Valadez G.J., García J.C., Zavala G.F. y Valle R.L. (2011). Producción de biomasa y concentración de azúcares de sorgo dulce en la región Huasteca Tamaulipeca. Rev. UDO Agr. 11, 58-70.

Walker R. (1990). Nitrates, nitrites and N-nitroso compounds: a review of the occurrence in food and diet, and toxicological implications. Food Addit. Contam. 7, 469-479. DOI: 10.1080/02652039009373938.

Wiedenfeld R.P. (1984). Nutrient requirements and use efficiency by sweet sorghum. Energy Agr. 3, 49-59. DOI: 10.1016/0167-5826(84)90004-4. 